\title{
Enhanced discrimination in autism
}

\author{
Michelle O'Riordan and Kate Plaisted \\ University of Cambridge, Cambridge, UK
}

\begin{abstract}
Children with autism are superior to typically developing children at visual search tasks (O'Riordan, Plaisted, Driver, \& Baron-Cohen, in press; Plaisted, O'Riordan, \& Baron-Cohen, 1998b). This study investigates the reasons for this phenomenon. The performance of children with autism and of typically developing children was compared on a series of visual search tasks to investigate two related problems. The first issue was whether the critical determinant of search rate in children is the discriminability of the display items, as it is in normal adults. The second question investigated was whether the superior performance of individuals with autism on visual search tasks is due to an enhanced ability to discriminate between display items. The results demonstrated that discriminability is the rate-determining factor for children with and without autism, replicating earlier findings with normal adults, and that children with autism have an enhanced ability to discriminate between display items. Thus, it seems that an enhanced ability to discriminate between display items underlies superior visual search in autism.
\end{abstract}

Autism is a pervasive developmental disorder characterized by symptoms that fall into three main areas (Wing \& Gould, 1979): abnormal social development, abnormal communicative development, and the presence of repetitive behaviour (American Psychiatric Association, APA, 1994; Baron-Cohen, 1988; Kanner, 1943; Rutter, 1983; Tager-Flusberg, 1985). Although the classic triad of impairments in autism is sufficient for a diagnosis (APA, 1987; World Health Organisation, WHO, 1992), abnormalities in visual processing are also characteristic of the disorder (Bryson, Wainwright-Sharpe, \& Smith, 1990; Hayes, 1987; Kanner, 1943; National Society for Autistic Children, NSAC, 1978; Rosenblum et al., 1980) and have long been documented (Kanner, 1943). For example, individuals with autism notice minor features of the environment that are overlooked by others, attend to particular stimuli to the exclusion of others, and notice minor changes to the environment that are indiscriminable to others (Hayes, 1987; NSAC, 1978). Recently, stimulus-processing abnormalities have been observed in autism in experimental studies. For example, individuals with autism show superior performance on the embedded-figures task compared to matched control groups

Requests for reprints should be sent to Michelle O'Riordan, Department of Experimental Psychology, University of Cambridge, Downing Street, Cambridge, CB2 3EB, UK. Email: mafo100@cus.cam.ac.uk

During the period of this work Michelle O'Riordan was supported by the MRC, and Kate Plaisted was supported by the Pinsent-Darwin Fund, University of Cambridge, and a Research Fellowship from St. John's College, Cambridge. We are grateful to the parents and teachers for allowing the authors to visit the schools to conduct this research and to the children for taking part. 
(Jolliffe \& Baron-Cohen, 1997; Shah \& Frith, 1983). In this task, the participant is asked to search for a "hidden" figure, which is embedded within a larger figure (Witkin, Oltman, Raskin, \& Karp, 1971).

Superior performance of children with autism compared to typically developing children has also been demonstrated in visual search tasks (O'Riordan, Plaisted, Driver, \& BaronCohen, in press; Plaisted, O'Riordan, \& Baron-Cohen, 1998b). In feature tasks, the participant is required to detect a target item that differs from all simultaneously presented distractor items along a single dimension (e.g., searching for a red $\mathrm{S}$ target among red $\mathrm{T}$ and green $\mathrm{X}$ distractors). In conjunctive tasks, the target shares one feature with one distractor type and another feature with the alternative distractor type (e.g., searching for a red $\mathrm{X}$ target among red $\mathrm{T}$ and green $\mathrm{X}$ distractors). Thus, in the conjunctive condition, the target is uniquely defined by the integration of its features (the colour red and the shape X). A common result in normal individuals is that, as the number of distractor items presented is increased (i.e., the display size increases), target detection time is slowed in conjunctive search tasks, but reaction time (RT) is independent of display size in feature search tasks (Duncan \& Humphreys, 1989; Treisman \& Gelade, 1980; Wolfe, Cave, \& Franzel, 1989). Studies have shown that children with autism were better than normally developing children, matched for age and general ability, at detecting a pre-specified target hidden among simultaneously presented distractors (O'Riordan et al., in press; Plaisted et al., 1998b). The performance of individuals with autism was superior to that of matched controls regardless of whether the target was uniquely defined by a feature or by a conjunction of features, except in easy tasks where the target and distractors were highly discriminable. Although these experiments demonstrated that the performance of individuals with autism on visual search tasks was superior to that of matched controls, they provided no explanation for this phenomenon. The experiments in this study attempt to elucidate the reasons for the superior performance of individuals with autism on search tasks.

Data on the mechanisms underlying normal visual search may help to identify the reasons for superior performance of individuals with autism on visual search tasks. One theory put forward to account for the typical profile of target detection times in feature and conjunctive search tasks in normal individuals was Feature Integration Theory (FIT-Treisman \& Gelade, 1980). This states that the visual display is initially represented in separate feature maps showing the distribution of individual features in the visual scene (i.e., a map for the colour red, one for lines, and another curves, etc.). Each item within a feature map is represented in its spatial location. In order for the visual attributes of each item to be combined to form multidimensional percepts, attention must be focused serially on each area of the visual field. Thus in feature tasks, where the target is uniquely defined by one feature, the target can simply be detected by the presence of activity in one feature map, and detection is rapid regardless of the number of simultaneously displayed distractor items. However, in the conjunctive task, where integration of feature maps is required for target detection, each item must be processed serially by a "spotlight" of focal attention. Hence, RT will increase as the number of items in the display increases. According to FIT, integration of the component parts of the object is the rate-determining factor in visual search tasks.

FIT states that in conjunctive search tasks, every item in the display must be examined location by location until the target is found. However, a second account for the standard profile of performance on feature and conjunction search tasks, by Wolfe et al. (1989), allows for 
the automatically processed information in the feature maps to guide the spotlight of attention to the locations where the combination of features defining the target is most likely to occur. According to this guided search model each item in the display is activated to the degree to which it shares features in common with a target. The spotlight of attention is guided to those locations with the highest summated level of activation in the display. However, in the case where the target and distractors share many features in common, many locations may be highly activated and hence search time will increase. In a feature search task, few features overall are held in common between target and distractors, and search will be fast (e.g., in a task in which the participant searches for a red $\mathrm{S}$ among green $\mathrm{X}$ and red $\mathrm{T}$ distractors, only colour is held in common, and only between one set of distractors and the target). Thus, the spotlight of attention can be guided with accuracy to the correct location of the target, and detection time will be fast. In contrast, in a conjunctive task, more features are held in common between the target and the distractors because the target shares a feature in common with each distractor type. As a result there will be more active locations in the visual display and therefore the spotlight will be guided with less accuracy to the target's location. Hence, detection time will be slow.

Thus, although both models state that conjunctive search tasks involve the integration of features, FIT holds that this process of integration is the rate-determining factor in conjunctive search tasks. By contrast, although the guided search model (Wolfe et al., 1989) regards integration as a necessary component of conjunctive target detection, it is the degree to which the target and distractors share features in common that is the rate-determining factor, and processes of feature integration are automatic.

There is a third model of visual search, the stimulus similarity model (Duncan \& Humphreys, 1989), which also supports the notion that the similarity of display items to one another is the critical determinant of search rate. This model suggests that items in the visual display are first grouped on the basis of physical similarity, then each group competes for access to visual short-term memory (VSTM) with those groups that are more similar to the target template, having a greater probability of access. Eventually the successful group gains access to VSTM, the group is searched, and the target is found. The stimulus similarity model and the guided search model make similar predictions, and it is suggested that these models are not necessarily mutually exclusive and may be successfully combined to provide a model of visual search (Duncan \& Humphreys, 1989). The phrase "discrimination models" will be used throughout this manuscript as a generic term for the guided search and stimulus similarity models.

It is possible to dissociate between the discrimination models and FIT as they make qualitatively different predictions. The discrimination models predict that the efficiency of target detection increases with increasing information being available from the parallel processing stage. For example, in a standard conjunctive search task each distractor is discounted as a target candidate by the presence of one feature. It is also possible to create a task where the target is uniquely defined by the combination of three features and where the presence of two features discounts each distractor as a target candidate. The discriminability models would predict faster target detection in this triple conjunction task than in the double conjunction task, as more information is available from the parallel stage in the triple task. FIT does not make such a prediction. If conjunction of features by attention is equally efficient across two or three features, then the standard feature integration theory would predict that the search slopes for 
the double and triple tasks would be comparable. Furthermore, if integration across three features is more effortful than integration across two features, feature integration theory would predict that search rate would be slower in the double task than in the triple task.

Experiments comparing simple double conjunctive target and triple conjunctive target (with target and distractors differing in terms of two features) detection in normal adults have found evidence to support the discriminability models (Quinlan \& Humphreys, 1987; Wolfe et al., 1989). It was found that triple conjunctions, when the target and each distractor shared one common feature, produced faster search rates than double conjunctions when the target shared only one feature with each distractor. Furthermore, RT to detect the triple conjunctive target under these conditions was independent of display size. This finding has since been replicated, and it is now well established that in normal adults discriminability of the items in the display is a critical factor determining search rate, and, although component features of items may initially be coded separately and subsequently integrated, it appears that the integration process is automatic.

From this discussion of the mechanisms underlying normal visual search it appears that discriminability of items in the display is the critical determinant of search rate. Therefore, one possible reason for the enhanced performance of children with autism, relative to typically developing children, is that children with autism have an enhanced ability to discriminate between items in the display. However, it is possible that the critical determinant of search rate in typically developing children and children with autism differs from that of normal adults, and this would undermine the notion that an enhanced discrimination ability underlies superior visual search in autism. Therefore, the main factor influencing visual search in normal children and in children with autism must be determined before differences in these mechanisms, between groups, can be investigated.

Thus, two issues were investigated in this study. The first question asked was whether typically developing children and children with autism show the same profile of performance as normal adults on the triple conjunctive search tasks described earlier. In other words, is discriminability the rate-determining factor in target detection tasks for normally developing children and children with autism? The second issue investigated was whether the superior performance of individuals with autism on visual search tasks is due to an enhanced ability to discriminate between items in the display.

\section{EXPERIMENT 1}

In Experiment 1 the performance of children with and without autism was compared on three search tasks. Task 1 was a double conjunctive search task in which only the combination of colour and orientation uniquely defined the target. Tasks 2 and 3 were triple conjunctive search tasks in which targets were only uniquely defined by the combination of three features-colour, size, and orientation - and these tasks varied from one another in the degree of targetdistractor similarity. The stimuli used in Experiment 1 are shown in Table 1.

The first comparison made was between Tasks 1 and 2. In Task 1, each distractor differed from the target by one feature, and in Task 2 each distractor differed from the target by two features. The tasks also differed in terms of the number of features that were required to uniquely define the target. The targets in Tasks 1 and 2 were uniquely defined by two and 
TABLE 1

Stimuli used in Experiment 1

\begin{tabular}{llccc}
\hline & & \multicolumn{3}{c}{ Distractor } \\
\cline { 3 - 4 } Task & Target & 1 & 2 & 3 \\
\hline 1 & & & \\
\hline
\end{tabular}

Note: Stimuli not drawn to size. Dimensions are given in Method section.

Black = red; grey $=$ green .

three features, respectively. If discriminability is the rate-determining factor in visual search for typically developing children then target detection time in this group will be faster in Task 2 than in Task 1, because of the greater discriminability between target and distractor in Task 2. Conversely, if integration is the principal determinant of search rate, performance should be comparable across tasks or perhaps even poorer in Task 2 than in Task 1. These predictions similarly hold for children with and without autism.

A second comparison was made between Tasks 2 and 3. These were both triple conjunctive tasks; however, whereas in Task 2 the distractors differed from the target by two features, in Task 3 they differed by one feature. Thus, although the number of features to be integrated was the same in both tasks, the tasks differed in degree of target-distractor discriminability. Target-distractor discriminability was higher in Task 2 than in Task 3. Once again, if typically developing children perform like normal adults they should be faster in Task 2 than in Task 3 . Furthermore, if children with autism are less affected by target-distractor similarity due to an enhanced discrimination ability, they should not be slowed to the same extent as typically developing children in Task 3 .

\section{Method}

\section{Participants}

Two groups of children participated: a group of 15 typically developing participants and a group of 15 children with autism. All children in the group with autism had been diagnosed by clinicians according to criteria such as those specified in DSM-IV (APA, 1994). In each group there were 12 male and 3 female participants. All children were assessed for general cognitive ability using Raven's Coloured Progressive Matrices (Raven, Court, \& Raven, 1990). Raven's matrices were chosen as the matching measure, as these are considered to be a non-linguistic measure of general ability and thus a conservative matching measure for this study, as children with autism characteristically have language impairments. There were no significant group differences in chronological age, $t(28)=-1.03, p=.31$, or Raven's Matrices scores, $t(28)=-1.14, p=.26$. These data are presented in Table 2 . 
TABLE 2

Chronological age and Raven's Matrices raw scores for children with autism and typically developing children in Experiment 1

\begin{tabular}{lcccccccc}
\hline & & \multicolumn{3}{c}{ Age $^{a}$} & & \multicolumn{3}{c}{ Raven's scores } \\
\cline { 3 - 5 } \cline { 7 - 9 } Group & $N$ & $M$ & $S D$ & Range & & $M$ & $S D$ & Range \\
\hline Control & 15 & $8: 7$ & $1: 8$ & $6: 9-11: 2$ & & 26 & 5 & $17-36$ \\
Autistic & 15 & $9: 2$ & $1: 1$ & $6: 9-11: 3$ & & 28 & 3 & $23-34$ \\
\hline
\end{tabular}

${ }^{\mathrm{a}}$ In years and months.

\section{Stimuli and apparatus}

The stimuli were generated by an Acorn RISC PC computer and presented on a 14-in. colour monitor. Participants were seated at a distance of $50 \mathrm{~cm}$ from the screen. Two keys, the " $Z$ " for negative trials and “.” for positive trials, were used for responding. The keyboard was covered by a black plastic cover to prevent other keys from being pressed.

Stimulus displays consisted of 4,16 , or 22 items arranged in a $14-\mathrm{cm}$ by 14 -cm imaginary grid, corresponding to a visual angle of $22.4^{\circ}$ centred on a central fixation point. The stimuli used for all four tasks were rectangles, which could vary along three dimensions of colour, size, and orientation and which are illustrated in Table 1 . The thick items measured $1.4 \mathrm{~cm}$ by $0.7 \mathrm{~cm}$, subtending approximately $1.6^{\circ}$ horizontally and $0.8^{\circ}$ vertically. The thin items measured $1.4 \mathrm{~cm}$. by $0.3 \mathrm{~cm}$, subtending approximately $1.6^{\circ}$ horizontally and $0.34^{\circ}$ vertically. The minimum distances between items were $1.4 \mathrm{~cm}$ between positions in a row and $1.4 \mathrm{~cm}$. between positions in a column. Display size refers to the number of items within the display and not the actual size of the area encompassed by these items.

\section{Design}

The experiment consisted of three search tasks. Each task contained two fully crossed factors: display size $(4,16$, or 22 items) and probe (target absent or target present) trials, which yielded six possible display types. There were 20 trials at each of these, yielding 120 trials per task. Trials were randomized within blocks of 30 trials for each search task, with equal representation of display size and target factors in each block. The first of these blocks was a practice block.

\section{Procedure}

Each participant was tested on three tasks, the presentation of which was counterbalanced between participants in each group. The participant was informed of the target to search for in that session and that certain keys were to be pressed depending on whether the target was present or absent. Prior to each task participants were given a block of practice trials involving the stimuli for that task, with the experimenter's instruction and assistance. Following these practice trials (immediately prior to the test trials) participants were instructed to respond as quickly as possible and with as few errors as possible.

On each trial the sequence of events was as follows: A fixation hash mark was presented on an otherwise blank screen for $500 \mathrm{~ms}$. The search display was then presented, at which point the timing was initiated. The search display remained on for $10 \mathrm{~s}$ or until the participant responded, whichever was the sooner. If the former occurred, the phrase "You were too slow" appeared in the centre of the screen for $500 \mathrm{~ms}$, followed by the presentation of the central hash for $500 \mathrm{~ms}$ indicating the onset of the next trial. If the correct response was made, the next trial was initiated. If an incorrect response was made, a tone sounded as an indication of the error. An incorrect trial was followed by a dummy trial, this was simply an additional trial in which the response was not recorded. This was to allow the participant to recover from 
an error. On the rare occasion that a button press occurred before the search display appeared on the screen the phrase "You pressed too soon!" was displayed at the centre of the screen for $500 \mathrm{~ms}$.

\section{Results and discussion}

For each participant RT data and error data were averaged for the trials for each particular combination of task ( 1 to 3 ), display size (4, 16, or 22 items), and probe (target present or target absent). Except where otherwise stated, a significance level of $p<.05$ was adopted for all statistical comparisons in this experiment and likewise for those that follow.

\section{Comparison 1}

$R T$ analysis. Figure 1 shows the mean RT scores for each group in Tasks 1 and 2. These scores were analysed by analysis of variance (ANOVA) with a between-subject factor of group (control or autistic) and three within-subject factors of task (1 or 2), probe (target present vs. target absent), and display size $(4,16$, or 22 items). The standard visual search effects were found in all the experiments but are not explicitly reported as they are not of major interest to the study. Details of these are available from the author on request.

Importantly, there was a main effect of task, $F(1,28)=130.31$, reflecting that Task 1 was more difficult than Task 2 . This result suggests that discriminability rather than integration is the critical determinant of search rate in children and replicates findings in normal adults. The interactions between task and display size, $F(2,56)=49.05$, between task and probe, $F(1,28)$ $=33.3$, and between task, probe, and display size, $F(2,56)=12.59$, provide further support for this notion. Target absent displays and increases in display size slowed responding to a greater extent in Task 1 than in Task 2, suggesting that Task 1 was more difficult.

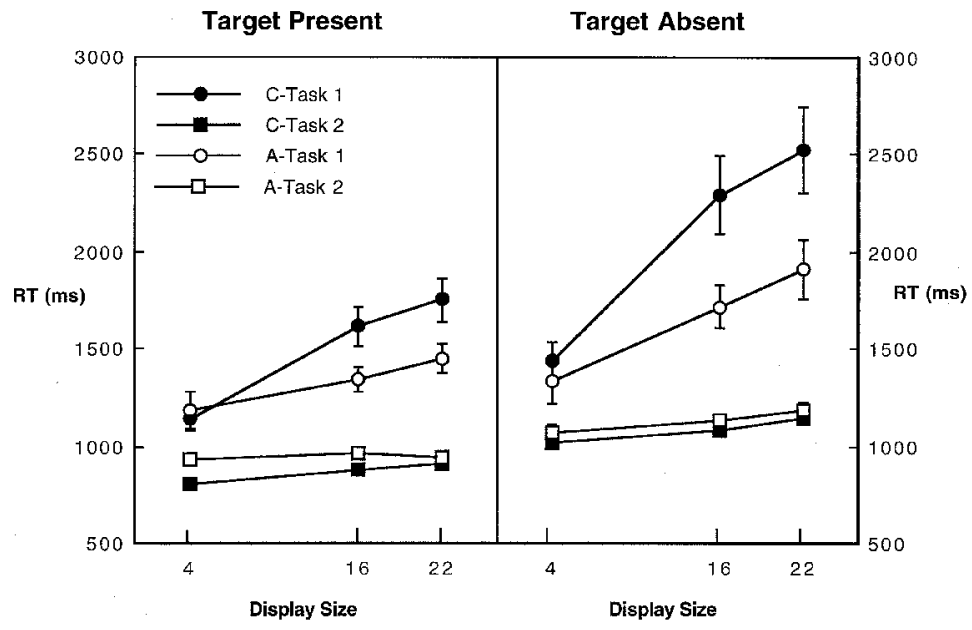

Figure 1. Results of Experiment 1: Comparison 1. The left-hand panel shows the mean RT data from target present trials for the control group (C) and the autistic group (A) in the double conjunctive and the triple conjunctive visual search tasks. The right-hand panel shows the data from the target absent trials. Each data point shows the mean $\mathrm{RT} \pm$ SEM. The autistic group was significantly faster than the control group in Task 2 . 
More interestingly, there was an interaction between group and task, $F(1,28)=11.13$. Simple effects of this interaction revealed that the children with autism were significantly faster than the normal group in Task $1, F(1,40)=5.51$, but not in Task $2(F<1)$. This replicates previous findings of superior visual search in autism in difficult (i.e., high targetdistractor similarity) tasks.

There was also a group by display size interaction, $F(2,56)=10.44$. Simple effects revealed that both groups of children were significantly slowed by an increase in display size, $F(2,56)=$ 79.08 , and $F(2,56)=19.21$, for the control and autistic groups, respectively, and there was no effect of group at any display size: $F<1, F(1,32)=2.14, p=.15$, and $F(1,32)=2.94, p=.10$, for display sizes of 4,16 , and 22, respectively. However, Figure 1 suggests that the group by display size interaction arose from a greater slowing in RT with increasing display size in the control than in the autistic group.

There were also three-way interactions between group, task, and probe, $F(1,28)=6.57$, and between group, task, and display size, $F(2,56)=6.87$. In order to determine the source of these interactions, separate ANOVAs were conducted on the data from each task. The source of the group by task by probe interaction was found to stem from the presence of a group by probe interaction in the Task 1 data, as reported in the previous comparison, but no such interaction in the data from Task $2(F<1)$. Furthermore, the group by task by display size interaction arose from the presence of a group by display size interaction in the data from Task 1 but not in the data from Task $2(F<1)$.

Error analysis. Error data were also analysed using the same factors as those in the initial analysis, in order to check that the observed RTs do not merely reflect group differences in speed-accuracy trade-off. Crucially this revealed that there was no difference between the number of errors made by the two groups, $F<1$, and no interactions involving the group term. Error rates were $3.5 \%$ and $3.9 \%$ for the control group and the autistic groups, respectively.

Comparison 1 established three important points. First, this comparison demonstrated faster RT in both groups in Task 1 than in Task 2, which suggests that the linear increase in $\mathrm{RT}$ with increases in display size in double conjunctive tasks results from target-distractor similarity rather than feature integration. This replicates in children those other studies that have considered the role of integration versus discriminability in adult visual search by Quinlan and Humphreys (1987) and Wolfe et al. (1989). In adults this pattern of results is taken to suggest that target-distractor discriminability is the rate-determining factor in visual search tasks. Thus, it appears that this is also the case for children with and without autism. Second, this comparison shows that, although children with autism showed the same pattern of RT as the typically developing children, they were faster in Task 1 . Taken together, these last two points suggest that there were differences between the two groups in the ability to discriminate between items. More specifically they suggest that the children with autism were better at discriminating between items than were the typically developing children. The third implication of this result is that processes of feature integration are automatic and intact in autism, a finding that lies in direct contradiction to the notion that individuals with autism have a perceptual integration impairment at the lowest level, which has been proposed by some (Frith, 1989; Frith \& Happé, 1994). 


\section{Comparison 2}

Comparison 1 suggests that superior visual search performance in autism results from an enhanced discrimination ability. However, it did not directly test the hypothesis of enhanced discrimination in autism because, although the two tasks differed in terms of target-distractor similarity, they also differed in terms of the number of features that were to be integrated to uniquely define the target. To directly examine the differences in discrimination ability between the two groups, a comparison was made between performance in Tasks 2 and 3 . These tasks differed only in terms of the number of features held in common between the target and the distractors.

RT analysis. The RT data are presented in Figure 2. The mean RT scores were analysed by ANOVA with the same factors as before. Importantly this revealed a significant main effect of task, $F(1,28)=59.01$. Performance was better in Task 2 than in Task 3 , thus demonstrating that increases in target-distractor similarity slowed visual search performance. Further evidence for this was the significant interactions between task and probe, $F(1,28)=12.03$, between task and display size, $F(2,56)=42.25$, between probe and display size, $F(2,56)=$ 16.42 , and between task, probe, and display size, $F(2,56)=10.08$. Increases in display size and target absent displays slowed responding to a greater extent in Task 3 than Task 2 , suggesting that Task 3 was more difficult.

More interestingly, this analysis revealed that the individuals with autism were overall significantly faster than were the control group, $F(1,28)=4.28$, and that the group by task interaction was significant, $F(1,28)=16.73$. Simple effects analysis revealed that although the autistic group was faster than the control group in Task $3, F(1,48)=15.58$, there was no difference between the RT of the two groups in Task $2(F<1)$. There was a group by display size

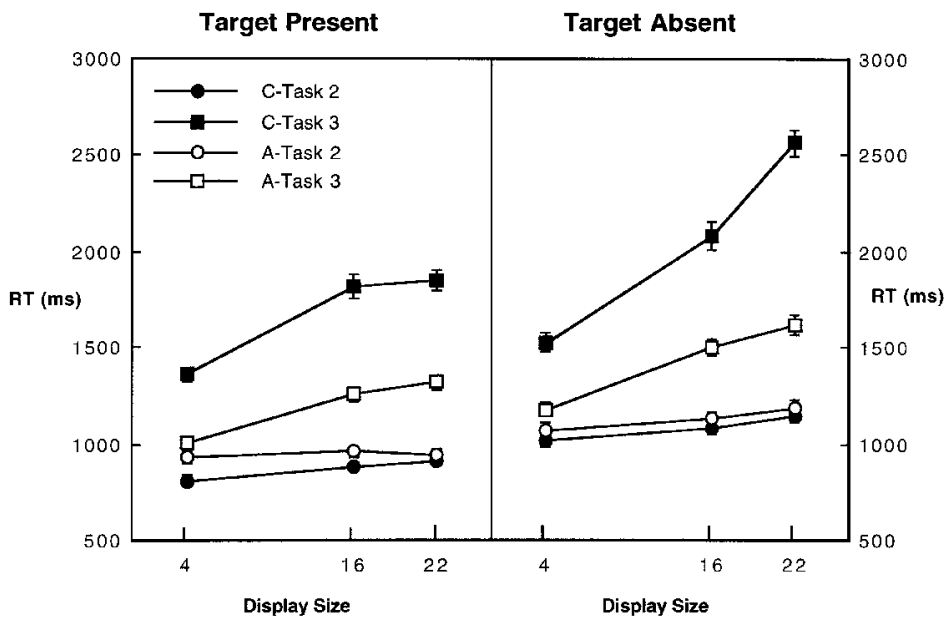

Figure 2. Results of Experiment 1: Comparison 1. The left-hand panel shows the mean RT data from target present trials for the control group $(\mathrm{C})$ and the autistic group (A) in Task C and Task D. The right-hand panel shows the data from the target absent trials. Each data point shows the mean RT \pm SEM. The autistic group was slowed significantly less than the control group by increases in target-distractor similarity. 
interaction, $F(2,56)=9.50$. Simple effects analysis revealed that the two groups were not performing differently for display sizes of 4 items, $F(1,31)=1.16, p=.29$, but that for display sizes of 16 items, $F(1,31)=4.30$, and 22 items, $F(1,31)=8.27$, the children with autism were faster than the typically developing children.

There were also interactions between group, task, and display size, $F(2,56)=4.85$, between group, probe, and display size, $F(2,56)=4.06$, and between group, task, probe, and display size, $F(2,56)=9.15$. In order to establish the sources of these interactions the data from each group were analysed separately using ANOVA with the same within-subject factors as in the original analysis.

This revealed that the group by probe by display size interaction stemmed from the presence of a probe by display size interaction in the data from the control group, $F(2,28)=27.05$, but not in the data from the children with autism, $F(2,28)=2.54, p=.0970$. Thus, whereas typically developing children were slowed by increases in display size to a greater extent in target absent than in target present trials, the children with autism were not affected differentially by display size in target present and target absent trials.

The four-way interaction between group, task, probe, and display size appeared to derive from the presence of a task by probe by display size interaction in the control, $F(2,28)=17.61$, but not in the autistic group $(F<1)$. Whereas in the control group there was a greater increase in RT with increasing display size for target absent than for target present trials, and the differential effect of display size on probe was greater in Task 3 than Task 2 for the control group, the same is not true for the autistic group.

The source of the three-way interaction between group, task, and display size was not identified from analysing the data from separate groups, task, or display sizes. However, the graph in Figure 2 suggests that although the effect of display size was greater in Task 3 than in Task 2 , in both groups, the difference between the effect of display size on the two tasks was greater in the control group than in the autistic group.

Error analysis. As before the error data were analysed by ANOVA with the same factors as those in the main analysis. Most importantly, there were no differences between the groups in terms of overall error rates $(F<1)$. Error rates were $4.0 \%$ and $4.9 \%$ for the control group and the autistic group, respectively.

The analysis of RT data therefore supports the conclusion drawn from Comparison 2, that target-distractor similarity is the critical rate-determining factor in search tasks in typically developing children and in children with autism, as target detection time is slowed under conditions of high target-distractor similarity. Most importantly, this comparison has supported the notion that individuals with autism are superior to normal children at discriminating between items, as although the children with autism were slowed by increases in targetdistractor similarity this group was slowed significantly less than the control group.

\section{Summary of Experiment 1}

In summary, these comparisons established that target-distractor similarity was the ratedetermining factor in these conjunctive visual search tasks, rather than feature integration (Wolfe et al., 1989) in typically developing children and in children with autism. That is, although these tasks clearly required feature integration (targets were uniquely defined only by 
integration of features), it was not the rate-determining factor. The demonstration that discriminability is the rate-determining factor in visual search tasks raises the possibility that the superior performance of individuals with autism on these tasks is due to an enhanced ability to discriminate. Comparison 2, which compared the performance of the children with and without autism on two triple conjunctive search tasks differing from one another in the degree of target-distractor similarity, explicitly tested this prediction. The results showed that the children with autism were not slowed to the same extent as the control children by an increase in target-distractor similarity and therefore supported the notion of a superior ability to discriminate between items in autism.

\section{EXPERIMENT 2}

Experiment 1 supported the notion that individuals with autism have a superior discrimination ability relative to typically developing individuals. Experiment 1 used manipulations of similarity between dimensions such that two or three dimensions could be shared between the target and surrounding distractors. In Experiment 2 the enhanced discrimination hypothesis was tested by using four conjunctive search tasks where similarity was manipulated within a dimension. The first task was a baseline task in which participants were asked to search for a red $\mathrm{X}$ target hidden among green $\mathrm{X}$ and red $\mathrm{C}$ distractors. The other tasks were constructed by modifying this first task to make the target less discriminable from the distractors in terms of colour or form or both. The stimuli from this experiment are drawn in Table 3. In the second task, where the target was a red $\mathrm{X}$ hidden among pink $\mathrm{X}$ and red $\mathrm{C}$ distractors, the target was less discriminable from the distractors in terms of colour. In the third task, where participants were required to search for a red $\mathrm{F}$ target among green $\mathrm{F}$ and red $\mathrm{E}$ distractors, the target and distractors were less discriminable than in the baseline task in terms of form. In the fourth task,

TABLE 3

Stimuli used in Experiment 2

Task

Note: Stimuli not drawn to size. Dimensions are given in Method section.

Black $=$ red; dark grey $=$ green; light grey $=$ pink. 
the target and distractors were more similar than in the baseline task in terms of both colour and shape. In this task, participants were required to search for a red $\mathrm{F}$ target hidden among pink $\mathrm{F}$ and red $\mathrm{E}$ distractors.

As discriminability is the critical factor influencing visual search task performance, it was predicted that the target detection time of the typically developing children and the children with autism would be slowed as target-distractor similarity increased. Further, it was predicted that, if individuals with autism have an enhanced ability to discriminate, then although the children with autism would be slowed by an increase in target-distractor similarity, the effect in this group would be reduced relative to that of the control group.

\section{Method}

\section{Participants}

Two groups of participants took part: A group of 13 children with autism and a group of 13 developmentally normal children. The children in the group with autism had been diagnosed according to the same criteria as before. There were 2 female and 11 male participants in each group. A total of 7 of the typically developing children and 8 of the children with autism in Experiment 2 had also participated in Experiment 1. For the subjects who participated in both the experiments the order of presentation of the experiments was randomized.

The children were assessed on their mental age using the Coloured Progressive Matrices (CPM), Sets A, Ab, and B, Raven et al. (1990). These data are presented in Table 4. Unpaired $t$-tests revealed that the chronological ages and the CPM raw scores of the two groups did not differ significantly, $t(24)=$ $0.23, p=.23$, and $t(24)=0.50, p=.69$, respectively, and therefore that the groups were matched for general ability.

\section{Apparatus and stimuli}

The apparatus used was the same as before. Each stimulus display consisted of 5, 15, or 25 elements (i.e., letters) arranged in an imaginary $16.8-\mathrm{cm}$ by $16.8-\mathrm{cm}$ grid (approximately $33^{\circ}$ visual angle) centred on a central fixation point (a hash). Each element measured $0.5 \mathrm{~cm}$ by $0.5 \mathrm{~cm}$, subtending approximately $1.0^{\circ}$ of visual angle horizontally and $1.0^{\circ}$ vertically. The minimum distances between elements in any display were $0.7 \mathrm{~cm}$ between positions in a row and $0.7 \mathrm{~cm}$ between positions in a column. Henceforth, the term display size refers to the number of elements in the display and not to the physical boundaries of the display, which remained fixed throughout. Display elements comprised two dimensions: colour (red, pink, and green) and form (C, E, F, T, and $\mathrm{X})$. The stimuli are drawn in Table 3.

TABLE 4

Chronological age and Raven's Matrices raw scores for children with autism and typically developing children in Experiment 2

\begin{tabular}{llccccccc}
\hline & & \multicolumn{3}{c}{ Age $^{a}$} & & \multicolumn{3}{c}{ Raven's scores } \\
\cline { 3 - 5 } \cline { 7 - 9 } Group & $N$ & $M$ & $S D$ & Range & & $M$ & $S D$ & Range \\
\hline Control & 13 & $8: 6$ & $1: 2$ & $7: 0-10: 0$ & & 30 & 5 & $17-35$ \\
Autistic & 13 & $9: 0$ & $1: 1$ & $6: 6-11: 0$ & & 28 & 3 & $23-35$ \\
\hline
\end{tabular}

${ }^{\mathrm{a}}$ In years and months. 


\section{Design and procedure}

The design and procedure were identical to those in Experiment 1 except that each task was for a double conjunctive target. The tasks differed from one another on the basis of the target and distractors and in the degree of target-distractor similarity (see Table 3).

\section{Results and discussion}

RT analysis. Figures 3 and 4 display the mean RT data for target present and target absent trials as a function of the factors of the experimental design. These RT scores were analysed by ANOVA with a between-subject factor of group (control or autistic) and three within-subject factors of task ( 1 to 4$)$, probe (target present or target absent), and display size $(5,15$, or 25 items). This analysis revealed a significant main effect of task, $F(3,24)=28.36$; NewmanKeuls pairwise comparisons revealed that RTs were significantly slower in Task 4 than in Tasks 1, 2, or 3, and slower in Tasks 2 and 3 than in Task 1 . However, there was no difference between the speed of responding in Tasks 2 and $3\left(\mathrm{RT}_{1}<\mathrm{RT}_{2,3}<\mathrm{RT}_{4}\right)$. Thus, increases in target-distractor similarity impaired performance. This notion was further supported by significant interactions between task and probe, $F(3,72)=15.25$, task and display size, $F(6,144)=$ 11.61 , and between task, probe, and display size, $F(6,144)=6.28$. Target absent displays and increases in display size slowed responding to a greater extent in tasks in which targetdistractor similarity was higher. These findings replicate standard findings in visual search experiments (Duncan \& Humphreys, 1989; Wolfe et al., 1989).

\section{Target Present Data}

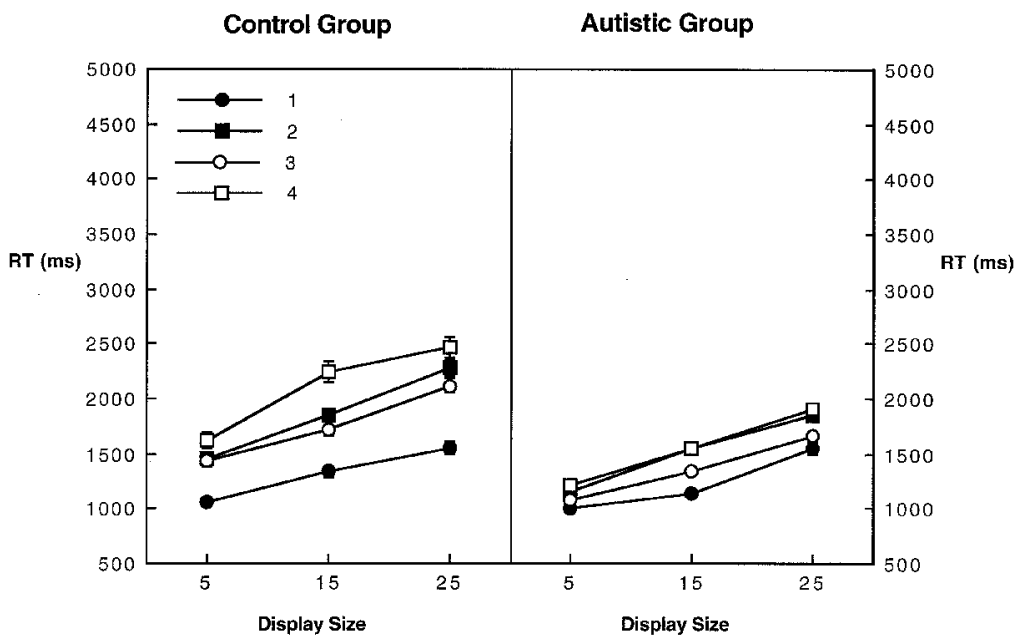

Figure 3. Results of the target present trials from Experiment 2. The left-hand panel shows the mean RT data for the control group, and the right-hand panel shows the data from the autistic group. Each data point shows the mean $\mathrm{RT} \pm \mathrm{SEM}$. Both groups were slowed by increases in target-distractor similarity but the autistic group was affected significantly less than the control group. 


\section{Target Absent Data}

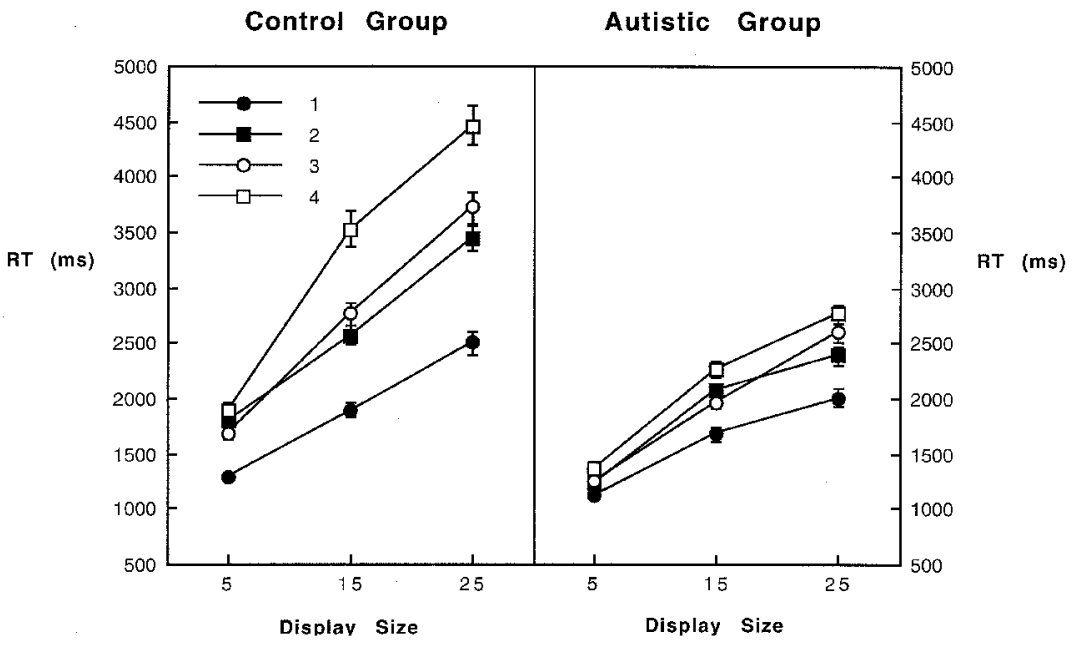

Figure 4. Results of the target absent trials from Experiment 2. The left-hand panel shows the mean RT data for the control group, and the right-hand panel shows the data from the autistic group. Each data point shows RT \pm SEM. Both groups were slowed by increases in target-distractor similarity but the autistic group were slowed significantly less than controls.

More interestingly, the analysis revealed a main effect of group, $F(1,24)=5.58$; RTs were faster in the autistic group than in the control group. Furthermore, the group by task interaction was significant, $F(3,72)=5.07$. Simple effects revealed that although there was no difference between the performance of the two groups in the first task $(F<1)$, the control group was significantly slower than the autistic group in the second, $F(1,34)=4.26$, third $F(1,34)=5.42$, and fourth task, $F(1,34)=11.61$.

The group by probe interaction was significant, $F(1,24)=7.93$. Simple effects revealed that although the autistic group was faster than the control group on target absent trials, $F(1$, $28)=9.33$, there was no difference between the two groups on target present trials, $F(1,28)=$ $2.19, p=.15$.

There was also a significant interaction between group and display size, $F(2,48)=4.55$. Simple effects revealed that the autistic group was significantly faster than the control group when searching a display of 25 items, $F(1,29)=9.43$, or 15 items, $F(1,29)=4.97$, but there was no difference between the two groups when searching 5 -item displays, $F(1,29)=2.03$, $p=.17$.

There were also three way interactions between group, task, and probe, $F(3,72)=3.78$, between group, task, and display size, $F(6,144)=2.56$, and between group, task, probe, and display size, $F(2,48)=6.62$. Furthermore, there was a four-way interaction between group, task, probe, and display size, $F(6,144)=2.44$.

To establish the source of these interactions the data from each condition were analysed separately, using ANOVA with a between-subject factor of group and within-subject factors of probe and display size. This revealed that although there was a group by probe interaction in 
the data from Task $2, F(1,24)=6.14$, Task $3, F(1,24)=5.93$, and Task $4, F(1,24)=9.21$, this was not the case in the data from Task $1, F(1,24)=2.35, p=.14$. This also showed that the group by condition by display size interaction resulted from there being a group by display size interaction in the data from Task $3, F(2,48)=3.19$, and Task $4, F(2,48)=5.91$, but not in the data from Task $1, F(2,48)=1.28, p=.29$, and Task $2, F(2,48)=2.93, p=.62$. Finally, these analyses revealed the source of the four-way interaction between group, task, probe, and display size; although there was an interaction between group, task, and display size in the data from Task $3, F(2,48)=3.47$, and Task $4, F(2,48)=8.48$, there was no such interaction in the data from Task $1, F(2,48)=2.56, p=.09$, or Task $2, F(2,48)=2.03, p=.14$. The source of the interaction between group, task, and display size was not revealed by analysing separately the data from each group, task, or display size, but Figures 3 and 4 suggest that this arose from the effect of task on display size (greater increases in display size in higher target-distractor similarity tasks) being greater in the control group than in the autistic group.

Error analysis. Error scores were analysed using ANOVA with the same factors as those in the initial RT analysis. Crucially, this revealed that there was no effect of group in the analysis of errors, $F<1$; the control group made $7.7 \%$ errors whereas the autistic group made $7.4 \%$ errors.

\section{Summary of Experiment 2}

As in Experiment 1 these results demonstrate that, although increasing target-distractor similarity retarded target detection time in both typically developing individuals and individuals with autism, the effect of increasing target-distractor similarity was smaller in the group of children with autism. This suggests that individuals with autism are superior at discriminating between items relative to matched controls. It is interesting to note that the group of individuals with autism were performing significantly faster than the control group in the second, third, and fourth tasks but not in the first task. This lack of superiority of the group of individuals with autism on a conjunctive task involving highly discriminable stimuli is interesting as it further supports the notion that the differential performance of individuals with and without autism on visual search tasks stems from differences in discriminability between groups. The level of superiority of the individuals with autism increases as target-distractor similarity increases and vice versa. Thus, the results from Experiment 2 support the hypothesis of superior discrimination in autism.

\section{GENERAL DISCUSSION}

These experiments have established that target-distractor similarity, rather than feature integration, is the rate-determining factor in conjunctive visual search tasks, in both children with and those without autism. That is, although these tasks clearly required feature integration, because targets were uniquely defined only by the integration of features, this process of integration was automatic, and it was not the rate-determining factor of search. This finding replicates in children the pattern of results found by Quinlan and Humphreys (1987) and Wolfe et al. (1989) in normal adults. These results are consistent with the idea that the profile of visual search performance (faster and reduced slowing with increasing display size on tasks where 
target-distractor similarity is low, and vice versa) remains constant from 6 months through to adulthood (Gerhardstein, 1997).

These experiments have also replicated previous findings that children with autism are faster than matched controls at visual search tasks (O'Riordan et al., in press; Plaisted et al., 1998b). The replication of superior performance of individuals with autism on conjunctive tasks, which require perceptual integration, together with the finding that processes of perceptual integration are automatic in autism is interesting because it contradicts the notion that there is a low-level perceptual integration deficit in autism (Frith, 1989).

The demonstration that discriminability was the rate-determining factor in visual search tasks, together with the superior performance of individuals with autism on these tasks, suggested that individuals with autism have an enhanced ability to discriminate between items. Comparison 2 of Experiment 1 and Experiment 2 explicitly tested this hypothesis by comparing the performance of the children, with and without autism, on search tasks that differed from one another in the degree of target-distractor similarity only. The results showed that the children with autism were not slowed to the same extent as the control children by an increase in target-distractor similarity and therefore supported the notion of superior discrimination in autism.

It could be argued that a reduced impact of manipulating target-distractor similarity on autistic relative to typical performance merely reflects that there is more room to see differences in visual search between groups in these more difficult tasks. In other words, perhaps children with autism are good at visual search per se, and this is merely more apparent in more difficult tasks. However, several factors suggest that enhanced discrimination ability in autism is the most probable explanation of the results presented here. First, the level of difference in visual search performance between individuals with and without autism has always been mirrored by target-distractor discriminability differences (O'Riordan et al., in press; Plaisted et al., 1998b). That is, in cases where there were no group differences in visual search performance the target and distractors were highly distinguishable. Second, if individuals with and without autism perform differently on visual search tasks then one or more of the processes involved in performance must be operating differentially in the two groups. From the discussion of the models of visual search in the Introduction it seems that integration, discrimination, grouping, and VSTM are all involved in visual search performance. It seems unlikely that perceptual integration operates more efficiently to give rise to superior visual search in autism because this has been demonstrated to be a rapid automatic process in both groups of children (Comparison 1, Experiment 1), and superiority of individuals with autism has been observed on feature search tasks in which integration is not a pre-requisite $\left(\mathrm{O}^{\prime} \mathrm{Riordan}\right.$ et al., in press). VSTM is another unlikely possibility as it has been established that working memory in children with autism is at best comparable to that of typically developing children (Russell, Jarrold, \& Henry, 1996) and may actually be worse (Bennetto, Pennington, \& Rogers, 1996). Thus, if anything, VSTM capacity in autism is likely to impair performance in visual search tasks. Differences in grouping between children with and without autism also seems unlikely. For example, if children with autism were to have weaker grouping mechanisms, the visual array would appear disordered with a low signal-to-noise ratio. Conversely, if grouping mechanisms were stronger in autism, the likelihood of the target being strongly grouped with distractors would increase, and thus target detection would be impeded. Both these possibilities predict worse rather than superior performance in autism. 
The final possibility, differential discrimination ability, appears to be the most likely process to underlie superior visual search in autism. It is widely accepted that discrimination ability is the critical determinant of search (Duncan \& Humphreys, 1989; Wolfe et al., 1989), and the size of the level of superiority of the autistic group on these search tasks is inversely related to the level of target-distractor similarity. Furthermore, there are also many examples of superior discrimination in autism outside the realms of visual search. For example, recent studies comparing the ability of individuals with and without autism to discriminate between novel, highly similar stimuli (Plaisted, O'Riordan, \& Baron-Cohen, 1998a; Plaisted \& O'Riordan, 1999) have shown that the individuals with autism were significantly better than matched controls.

It is interesting that the superiority of the group of individuals with autism is often confined to their performance on target absent trials. This phenomenon may also be consistent with the notion that superior visual search in autism stems from an enhanced discrimination ability. If the target is easily discriminable from distractors and thus highly salient when present to the individual with autism then on target absent trials the lack of a highly salient item will also be highly apparent and thus quickly and confidently indicated. However, if the target's presence were less salient to a typically developing individual then on target absent trials they may be less confident and consequently carefully check the display before responding. Hence the difference between the two groups might be exaggerated on target absent trials.

The experiments in this study have provided evidence that superior visual search in autism results from an enhanced discrimination ability but they do not specify the reasons for this superior discrimination. Several processes may underlie the ability to discriminate between items in the visual scene (Wolfe et al., 1989) and it is possible to divide these processes into two broad categories. First, superior discrimination could result from differences in bottom-up perceptual processing such that two items appear to be more distinct to the individual with autism. That is, individuals with autism could have reduced perception of similarity and thus an augmented perception of difference.

The second possible explanation for the superior ability of individuals with autism to discriminate between items in the display is that top-down mechanisms of excitation and inhibition acting on representations of search items (top-down modulation of representation-activation) are operating differently in autism such that non-target features are inhibited to a greater extent, and/or target features are excited to a greater extent. Such a difference could provide an alternative account for superior search performance that does not appeal to the potential differential that early perceptual processes suggested previously. More specifically, the activation level for the target item would be more distinct from that for distractor items under these conditions, and thus the target would be detected more easily. Early work suggests that enhanced discrimination in autism does not result from differential mechanisms of topdown modulation of activation of stimulus representations (O'Riordan, 2000) but future research will be required to demonstrate that differential early perceptual processes underlie enhanced discrimination in autism.

This work suggests that individuals with autism have an enhanced ability to discriminate between items and that this difference in processing underlies their superior performance on visual search tasks. Furthermore, an enhanced discrimination ability could relate to many of the accounts of disturbed visual processing in autism, such as superior performance on the embedded figures task (Jolliffe \& Baron-Cohen, 1997; Shah \& Frith, 1983), in which a target 
shape must be discriminated from the distractor shapes, which are all embedded within a larger picture (Witkin, 1964). Enhanced discrimination in autism could also be linked to their superior block design performance (Rumsey \& Hamberger, 1988; Shah \& Frith, 1993). In this task, participants must construct a pattern from blocks with variously patterned faces (Kohs, 1923), and an enhanced ability to discriminate between the block faces could facilitate performance. Thus, an enhanced discrimination ability could be related to many of the visuo-perceptual anomalies characteristic of autism but such a disturbance could have implications far beyond these perceptual/attentional abnormalities. Indeed, any disturbance in stimulus processing would fundamentally alter the quality of the information that the child receives from the environment and would have profound effects on psychological processing and development. For example, an enhanced discrimination ability in autism would reduce the amount of information available across which to generalize from one situation to another (Plaisted et al., 1998a). The ability to generalize is essential to many psychological processes, such as inferential and analogical reasoning and categorization, and therefore we would expect the product of these processes to be compromised in autism, because the input is impoverished as a result of the enhanced discrimination ability. In turn, these processes are essential for normal cognitive, social, and emotional development and it is across these domains of development that individuals with autism are characteristically impaired.

\section{REFERENCES}

American Psychiatric Association. (1987). Diagnostic and statistical manual of mental disorders (3rd ed.). Washington, DC: Author.

American Psychiatric Association. (1994). Diagnostic and statistical manual of mental disorders (4th ed.). Washington, DC: Author.

Baron-Cohen, S. (1988). Without a theory of mind one cannot participate in a conversation. Cognition, 29, 83-84.

Bennetto, L., Pennington, B., \& Rogers, S. (1996). Intact and impaired memory functions in autism. Child Development, 67, 1816-1835.

Bryson, S.E., Wain wright-Sharpe, J.A., \& Smith, I.M. (1990). Autism: A developmental neglect syndrome? In J.T. Enns (Ed.), The development of attention: Research and theory. Amsterdam: North-Holland.

Duncan, J., \& Humphreys, G.W. (1989). Visual-search and stimulus similarity. Psychological Reviem, 96, 433-458.

Frith, U. (1989). Autism: Explaining the enigma. Oxford: Basil Blackwell.

Frith, U., \& Happé, F. (1994). Autism: Beyond "theory of mind". Cognition, 50, 115-132.

Gerhardstein, P. (1997). Reaction-time measures of visual search at 1, 2 and 3 years of age. Poster presented at the Annual Meeting of the Society for Research in Child Development, Washington, DC, USA.

Hayes, R. (1987). Training for work. In D.J. Cohen \& P.M. Donnellan (Eds.), Handbook of autism and pervasive developmental disorders. Silver Springs, MD: Winston.

Jolliffe, T., \& Baron-Cohen, S. (1997). Are people with autism or Asperger's Syndrome faster than normal on the Embedded Figures Task? Journal of Child Psychology and Psychiatry, 38, 527-534.

Kanner, L. (1943). Autistic disturbance of affective contact. Nervous Child, 2, 217-250.

Kohs, S.C. (1923). Intelligence measurement. New York: Macmillan.

National Society for Autistic Children. (1978). National Society for Autistic Children definition of the syndrome of autism. Fournal of Autism and Developmental Disorders, 8, 162-167.

O'Riordan, M.A. (2000). Superior Modulation of activation levels of stimulus representations does not underlie superior discrimination in autism. Cognition, 77, 81-96.

O'Riordan, M.A, Plaisted, K., Driver, J., \& Baron-Cohen, S. (in press). Superior visual search in autism. Journal of Experimental Psychology: Human Perception and Performance.

Plaisted, K., O'Riordan, M.A., \& Baron-Cohen, S. (1998a). Enhanced discrimination of novel, highly similar stimuli by adults with autism during a perceptual learning task. Fournalof Child Psychology and Psychiatry, 39, 765-775. 
Plaisted, K., O'Riordan, M.A., \& Baron-Cohen, S. (1998b). Enhanced visual search for a conjunctive target in autism. A research note. Fournal of Child Psychology and Psychiatry, 39, 777-783.

Plaisted, K.C., \& O'Riordan, M.A. (1999). Autistic children show enhanced perceptual learning-further evidence of reduced generalisation in autism. Manuscript in preparation.

Quinlan, P.T., \& Humphreys, G.W. (1987). Visual-search for targets defined by combinations of colour, shape, and size - an examination of the task constraints on feature and conjunction searches. Perception $E$ P Psychophysics, $41(5), 455-472$.

Raven, J.C., Court, J.H., \& Raven, J. (1990). Coloured Progressive Matrices. Oxford: Oxford Psychologists Press.

Rosenblum, S.M., Arick, J.R., Krug, D.A., Stubbs, E.G., Young, N.B., \& Pelson, R.O. (1980). Auditory brainstem evoked responses in autistic children. Fournal of Autism and Developmental Disorders, 10, 215-225.

Rumsey, J., \& Hamberger, S. (1988). Neuropsychological findings in high functioning men with infantile autism, residual state. Fournal of Clinical and Experimental Neuropsychology, 10, 201-221.

Russell, J., Jarrold, C., \& Henry, L. (1996). Working memory in children with autism and with moderate learning difficulties. Fournal of Child Psychology and Psychiatry, 37, 673-686.

Rutter, M. (1983). Cognitive deficits in the pathogenesis of autism. Fournal of Child Psychology and Psychiatry, 24, $513-531$.

Shah, A., \& Frith, U. (1983). An islet of ability in autism: A research note. Fournal of Child Psychology and Psychiatry, 24, 613-620.

Shah, A., \& Frith, U. (1993). Why do autistic individuals show superior performance on the block design test? Fournal of Child Psychology and Psychiatry, 34, 1351-1364.

Tager-Flusberg, H. (1985). Psycholinguistic approaches to language and communication in autism. In E. Schopler \& G.B. Mesibov (Eds.), Communication problems in autism. New York: Plenum Press.

Treisman, A., \& Gelade, G. (1980). A feature integration theory of attention. Cognitive Psychology, 12, 97-136,

Wing, L., \& Gould, J. (1979). Severe impairments of social interaction and associated abnormalities in children: Epidemiology and classification. Fournal of Autism and Developmental Disorders, 9, 11-29.

Witkin, H., Oltman, P., Raskin, E., \& Karp, S. (1971). A manualfor the Embedded Figures Test. Palo Alto, CA: Consulting Psychologists Press, Inc.

Witkin, H.A. (1964). Origins of cognitive style. New York: Harper \& Row.

Wolfe, J.M., Cave, K.R., \& Franzel, S.L. (1989). A modified feature integration model for visual search. Fournal of Experimental Psychology: Human Perception and Performance, 15, 419-433.

World Health Organisation . (1992). World Health Organisation: International Classification of diseases and related health problems (ICD-10)(10th ed.). Geneva: World Health Organisation. 